OPEN ACCESS

Edited by:

David Perrais,

Centre National de la Recherche Scientifique (CNRS), France

Reviewed by: Sandra Jurado, Instituto de Neurociencias de Alicante (IN), Spain Andrew Charles Penn, University of Sussex, United Kingdom

${ }^{*}$ Correspondence: Mikyoung Park mpark@kist.re.kr; mikyoungpark7@gmail.com

Received: 27 July 2018 Accepted: 25 September 2018 Published: 11 October 2018

Citation: Park M (2018) AMPA Receptor Trafficking for Postsynaptic Potentiation.

Front. Cell. Neurosci. 12:361. doi: 10.3389/fncel.2018.00361

\section{AMPA Receptor Trafficking for Postsynaptic Potentiation}

\author{
Mikyoung Park ${ }^{1,2 *}$ \\ ${ }^{1}$ Center for Functional Connectomics, Brain Science Institute, Korea Institute of Science and Technology (KIST), Seoul, \\ South Korea, ${ }^{2}$ Department of Neuroscience, Korea University of Science and Technology, Daejeon, South Korea
}

Long-term potentiation (LTP) of excitatory synaptic strength, which has long been considered a synaptic correlate for learning and memory, requires a fast recruitment of additional $\alpha$-amino-3-hydroxy-5-methyl-4-isoxazolepropionate (AMPA) receptors (AMPARs) to the postsynaptic sites. As cell biological concepts have been applied to the field and genetic manipulation and microscopic imaging technologies have been advanced, visualization of the trafficking of AMPARs to synapses for LTP has been investigated intensively over the last decade. Recycling endosomes have been reported as intracellular storage organelles to supply AMPARs for LTP through the endocytic recycling pathway. In addition, exocytic domains in the spine plasma membrane, where AMPARs are inserted from the intracellular compartment, and nanodomains, where diffusing AMPARs are trapped and immobilized inside synapses for LTP, have been described. Furthermore, cell surface lateral diffusion of AMPARs from extrasynaptic to synaptic sites has been reported as a key step for AMPAR location to the synaptic sites for LTP. This review article will discuss recent findings and views on the reservoir(s) of AMPARs and their trafficking for LTP expression by focusing on the exocytosis and lateral diffusion of AMPARs, and provide some future directions that need to be addressed in the field of LTP.

Keywords: AMPA receptors, long-term potentiation, postsynapse, exocytosis, lateral diffusion

\section{INTRODUCTION}

Synapses are fundamental units of brain function and possess the remarkable ability to change their strength in function and structure through synaptic plasticity. Long-term potentiation (LTP), a well characterized form of synaptic plasticity that has long been considered a synaptic correlate for learning and memory, was discovered in the hippocampus in 1973 (Bliss and L $\phi$ mo, 1973). Prior to the discovery of LTP, Hebb's (1949) postulate that learning and memory involves synaptic strengthening elicited by the coordinated firing of pre- and postsynaptic cells was suggested. In addition, beginning with Ramón y Cajal (1852-1934), many neuroscientists have suggested that learning and memory should involve synaptic modifications (Malenka, 2003). A type of glutamate receptor, the $\alpha$-amino-3-hydroxy-5-methyl-4-isoxazolepropionate (AMPA) receptor (AMPAR), has been investigated intensively as a key player in synaptic modifications involved in synaptic transmission, synaptic plasticity and, ultimately, learning and memory. LTP and long-term depression (LTD), another well characterized form of synaptic plasticity in the hippocampus, are expressed by long lasting changes of AMPARmediated synaptic responses. Exocytosis and endocytosis of AMPARs play critical roles in LTP and LTD, respectively, in aspects of both functional and structural plasticity of synapses 
(Kessels and Malinow, 2009; Anggono and Huganir, 2012; Huganir and Nicoll, 2013). Indeed, learning induces LTP in the hippocampus (Whitlock et al., 2006). Learning alters AMPAR phosphorylation and synaptic delivery of AMPARs (Whitlock et al., 2006), which are readouts for LTP (Heynen et al., 2000; Lee et al., 2000; Malenka, 2003). Although studies of LTP were conducted originally through electrophysiological approaches, advances in the tools of molecular and cellular biology, biochemistry, state-of-the-art imaging and genetics have provided much more sophisticated information of AMPAR trafficking to synapses to support LTP mechanisms. This review article provides a brief introduction of AMPARs and LTP, followed by a focus on recent findings and views on AMPAR reservoir(s) for LTP by examining studies on the exocytosis and cell surface lateral diffusion of AMPARs during LTP.

\section{AMPARs AND LTP}

AMPARs are major ionotropic glutamate receptors that respond to physiological glutamate, a major excitatory neurotransmitter in the mammalian central nervous system. AMPARs have four subunits, GluA1-GluA4 encoded by Gria1-Gria4 genes, and those subunits form hetero-tetramers composed of two dimers (Wisden and Seeburg, 1993; Hollmann and Heinemann, 1994; Dingledine et al., 1999; Traynelis et al., 2010; Chater and Goda, 2014). The combination of each subunit forms a developmentally distinct receptor complex in the hippocampus (Wenthold et al., 1996; Zhu et al., 2000). Immature hippocampal neurons at early developmental stages express the GluA4 subunit, which complexes with the GluA2 subunit (Zhu et al., 2000). However, mature hippocampal neurons express two predominant combinations of AMPAR subunits, GluA1/GluA2 or GluA2/GluA3 heterotetrameric receptors (Wenthold et al., 1996). Regulation of the precise localization and number of AMPARs at the cell surface membrane is critical for most excitatory synaptic transmission at the steady state and also for long-term synaptic plasticity, such as LTP and LTD (Song and Huganir, 2002; Bredt and Nicoll, 2003).

The majority of studies on LTP have been performed on excitatory synapses between Schaffer collateral-commissural axons and CA1 pyramidal neuron dendrites in the hippocampus (Bear and Kirkwood, 1993; Kirkwood et al., 1993; Nicoll and Roche, 2013). While LTP is triggered rapidly by a brief high-frequency stimulation (HFS), it persists for days or even weeks in vivo. The "early phase" of LTP, which lasts approximately $60 \mathrm{~min}$, requires the activation of $\mathrm{N}$-methyl-D-aspartate (NMDA) receptors (NMDARs) for its induction, together with subsequent $\mathrm{Ca}^{2+}$ influx and calcium/calmodulin-dependent protein kinase II (CaMKII) activation (Malenka et al., 1989; Malinow et al., 1989; Silva et al., 1992; Lisman, 1994; Lisman et al., 1997; Malenka and Nicoll, 1999). In addition, delivery of new AMPARs to the postsynaptic sites is believed to be responsible for LTP expression in its early phases. One distinguishable characteristic of the "late phase" of LTP, which lasts days or even weeks, from the "early phase" of LTP, is that the late phase requires gene transcription and new protein synthesis
(Schuman et al., 2006; Reymann and Frey, 2007; Johnstone and Raymond, 2011; but also see Abbas et al., 2009; Villers et al., 2012).

Three major questions have been the focus of studies in the field of LTP. First, studies examined whether the increase in synaptic strength during LTP at Schaffer collateral-CA1 synapses is due primarily to presynaptic or postsynaptic modifications (Kullmann and Siegelbaum, 1995; Nicoll and Malenka, 1995; Emptage et al., 1999, 2003; Malenka and Nicoll, 1999; Ward et al., 2006; Kerchner and Nicoll, 2008; Enoki et al., 2009; Kullmann, 2012; Chater and Goda, 2014; Granger and Nicoll, 2014; Padamsey and Emptage, 2014). Second, studies have been conducted to determine which AMPAR subunits are responsible for LTP expression (Jia et al., 1996; Zamanillo et al., 1999; Hayashi et al., 2000; Shi et al., 2001; Granger et al., 2013; Granger and Nicoll, 2014; Diaz-Alonso et al., 2017; Zhou et al., 2018). Finally, studies have examined whether AMPARs are located to the synapse for LTP expression through exocytosis and/or lateral diffusion. The debate about whether the location of LTP expression at CA1 synapses is presynaptic or postsynaptic has lasted for more than two decades. Currently, most data, even those previously supporting a presynaptic change of increased release probability or decreased synaptic failure in LTP expression can be reconciled with postsynaptic changes by the "silent synapse" concept (Isaac et al., 1995, 1996; Liao et al., 1995, 1999; Durand et al., 1996; Gomperts et al., 1998; Nusser et al., 1998; Petralia et al., 1999; Takumi et al., 1999; Kerchner and Nicoll, 2008). It is now generally accepted that LTP expression at Schaffer collateral-CA1 synapses is mediated by AMPAR insertion into the synapse, supporting the postsynaptic view for LTP expression (Malenka and Nicoll, 1999; Shi et al., 1999; Hayashi et al., 2000; Malinow and Malenka, 2002; Song and Huganir, 2002; Bredt and Nicoll, 2003; Nicoll, 2003; Chater and Goda, 2014; Granger and Nicoll, 2014). Single channel conductance increases of AMPARs have been suggested to mediate LTP expression in the CA1 region of the hippocampus (Benke et al., 1998). However, a recent reevaluation of this study by the same group showed that insertion of AMPARs with high conductance can account for LTP expression (Benke and Traynelis, 2018). In addition to postsynaptically expressed LTP at Schaffer collateral-CA1 synapses, a distinct form of LTP at mossy fiber synapses, which is independent of NMDARs and expressed presynaptically unlike that at Schaffer collateral-CA1 synapses, has been well investigated and extensivley discussed (Nicoll and Malenka, 1995; Nicoll and Schmitz, 2005; Granger and Nicoll, 2014). AMPARs, particularly those containing the GluA1 subunit, have been suggested to play an important role in LTP expression at CA1 synapses in studies using knockout mice lacking GluA1 or GluA2 and electrophysiological recordings of hippocampal slice expressing tagged GluA1 or GluA2 (Jia et al., 1996; Zamanillo et al., 1999; Hayashi et al., 2000; Shi et al., 2001; Diaz-Alonso et al., 2017). The GluA1 subunit requirement for LTP has been investigated and supported by studies focusing on the cytoplasmic carboxy terminal (C-terminal) tail, which has been demonstrated to be involved in intracellular signaling through phosphorylation, palmitoylation or protein interactions (Barria et al., 1997; 
Hayashi et al., 2000; Shi et al., 2001; Esteban et al., 2003; Lee et al., 2003; Boehm et al., 2006; Lin et al., 2009). However, the GluA1 C-terminal tail requirement for LTP was challenged by a report showing that LTP requires AMPAR trafficking, independent of subunit type (Granger et al., 2013). Interestingly, recent studies have demonstrated that the extracellular aminoterminal domain (ATD) of AMPARs governs their trafficking for synaptic plasticity dependent on the AMPAR subunit type (Diaz-Alonso et al., 2017; Watson et al., 2017). Further, the spatial resolution of AMPARs delivered into the synapse for LTP has been questioned whether it is through exocytosis directly from the intracellular pool to synaptic sites or through lateral mobility from the extrasynaptic plasma membrane or a combination of each (Lledo et al., 1998; Lu et al., 2001; Park et al., 2004; Kopec et al., 2006, 2007b; Yudowski et al., 2007; Jaskolski and Henley, 2009; Lin et al., 2009; Makino and Malinow, 2009; Petrini et al., 2009; Kennedy et al., 2010; Opazo et al., 2010; Patterson et al., 2010; Cho et al., 2015; Penn et al., 2017; Temkin et al., 2017; Wu et al., 2017).

\section{EXOCYTOSIS AND LATERAL MOBILITY OF AMPARs FOR LTP}

Much evidence suggests that LTP expression is mediated by postsynaptic mechanisms (Lisman et al., 2012; Lu and Roche, 2012; Granger et al., 2013; Granger and Nicoll, 2014) and requires exocytosis at or near the postsynaptic membrane, which results in an increase in the number of AMPARs (Lledo et al., 1998; Lu et al., 2001; Park et al., 2004; Patterson et al., 2010; Ehlers, 2013; Wu et al., 2017). Initial studies showed that introducing various reagents that disrupt membrane fusion into the postsynaptic cells blocks LTP at Schaffer collateral-CA1 synapses (Lledo et al., 1998), implying that the exocytosis of intracellular vesicles harboring AMPARs is an essential step for LTP. A serial electron microscopy study demonstrated that endosomal compartments are located in dendrites and dendritic spines and serve as intracellular storehouses for the plasma membrane (Cooney et al., 2002). Some molecules that are retained on endosomal compartments can be delivered rapidly to the cell surface in response to stimuli (Lampson et al., 2001; Bryant et al., 2002; Cooney et al., 2002; Zeigerer et al., 2002; Govers et al., 2004; Guilherme et al., 2004). In addition, another study showed that synaptic activity that can induce LTP drives AMPARs to be endocytosed and reinserted to the plasma membrane (Ehlers, 2000). Together, these data suggest that endosomal organelles involved in endocytic recycling transport can serve as primary intracellular membrane compartments mobilized to the plasma membrane in response to LTP-inducing stimuli (Ehlers, 2013). Indeed, disrupting the transport of recycling endosomes to the plasma membrane using dominant negative forms of Rab11 (Ullrich et al., 1996; Zerial and Mcbride, 2001), syntaxin 13 (Prekeris et al., 1998), or Eps15 homology domain protein Rme1/EHD1 (Grant et al., 2001; Lin et al., 2001) blocked synaptic delivery of AMPARs during LTP (Park et al., 2004). Postsynaptic synaptotagmin-1 and synaptotagmin-7 were reported to mediate
GluA1 exocytosis during LTP by acting as postsynaptic $\mathrm{Ca}^{2+}$. sensors (Wu et al., 2017). Complexin, a regulator of SNAREmediated neurotransmitter release in presynapses (Reim et al., 2001; Maximov et al., 2009), binds to SNARE complexes to mediate AMPAR exocytosis during LTP in postsynapses (Ahmad et al., 2012). Subsequently, postsynaptic SNARE proteins such as syntaxin 3, SNAP-47 and synaptobrevin-2, which are distinct from proteins involved in presynaptic neurotransmitter release, were reported to regulate AMPAR exocytosis during LTP (Jurado et al., 2013).

Visualization of activity-triggered exocytosis of AMPARs in dendrites and dendritic spines is possible using the $\mathrm{pH}$-sensitive superecliptic pHluorin (SEP), whose fluorescence is quenched at low pH (Miesenbock et al., 1998). Indeed, using SEP-tagged AMPARs, the postsynaptic exocytosis of AMPARs during LTP has been visualized directly (Kopec et al., 2006, 2007a; Yudowski et al., 2007; Lin et al., 2009; Makino and Malinow, 2009; Petrini et al., 2009; Araki et al., 2010; Kennedy et al., 2010; Patterson et al., 2010; Cho et al., 2015). Glycine-induced LTP has been shown to be mediated by an accumulation and immobilization of SEP-GluA1s at synapses, due to both exocytosis and stabilization of GluA1s at the postsynaptic density (PSD; Petrini et al., 2009). Impairment of GluA1 recycling exocytosis with a dominantnegative mutant of Rab11 results in GluA1 being less mobile at synapses. In addition, the displacement of endocytic zones from the PSD by a point mutant of dynamin-3 unable to bind Homer1 (Lu et al., 2007) impairs glycine-induced LTP expression by blocking GluA1 recycling (Petrini et al., 2009). Taken together, these results suggest that the GluA1 endocytic recycling pool is crucial for maintaining a mobile population of surface GluA1s that can be mobilized to synapses for LTP. Bath application of glycine for inducing LTP increases the exocytic events of SEP-GluA1 in dendrites and dendritic spines (Yudowski et al., 2007; Cho et al., 2015). In a more localized activation using two-photon glutamate uncaging, which mimics single synaptic release with sufficient spatiotemporal resolution (Matsuzaki et al., 2004; Bagal et al., 2005; Harvey and Svoboda, 2007; Lee et al., 2009), SEP-GluA1 was observed to be exocytosed to dendrites and activated spines (Makino and Malinow, 2009; Patterson et al., 2010). The increase of AMPARmediated currents was observed in spines initially, and then in the dendrite following glutamate uncaging-evoked LTP (Makino and Malinow, 2009), consistent with GluA1 insertion directly to the spines.

Many studies have supported the idea that LTP triggers the exocytosis of AMPARs required for expression. In addition, several studies have demonstrated how the intracellular recycling endosome that stores AMPARs is mobilized to near or at the spine for LTP, and where AMPARs are exocytosed to the spine surface in relation to the PSD for LTP (Wang et al., 2008; Kennedy et al., 2010). The actin-based $\mathrm{Ca}^{2+}$-sensitive motor protein myosin $\mathrm{Vb}$ has been reported to mediate the translocation of recycling endosomes harboring AMPARs into spines during LTP (Wang et al., 2008). Blockade of myosin Vb using RNA interference or chemical-genetic inhibition results in reduced LTP-induced SEP-GluA1 insertion and hippocampal slice LTP, indicating that the myosin Vb-mediated mobilization 
of recycling endosomes is required for synaptic potentiation (Wang et al., 2008). Related actin-based myosin Va has also been reported to mediate the translocation of AMPARs to spines from the dendritic shaft during LTP (Correia et al., 2008). Neurons expressing a dominant-negative form of myosin Va or a short interfering RNA specific for myosin Va showed a blockade of synaptic delivery of GluA1 and LTP (Correia et al., 2008). Conversely, myosin Va mutant mice showed normal synaptic plasticity (Schnell and Nicoll, 2001), suggesting a potential compensation by other Class $\mathrm{V}$ myosins. In a recent report on another actin-dependent motor protein myosin IXa, myosin IXa ${ }^{+/-}$mice displayed impaired LTP (Folci et al., 2016), together indicating that myosin motor proteins play roles in AMPAR delivery during LTP. Using SEP-GluA1 and transferrin receptor (TfR), a classic recycling endosomal marker, GluA1 in TfR-positive recycling endosomes was shown to be exocytosed to spines adjacent to the PSD during glycine-induced LTP. Newly inserted SEP-GluA1 either quickly diffuses out of the spine or stays near the site of fusion in spines, whereas TfRs that co-exocytosed with SEP-GluA1 always diffused out of the spine immediately following the co-appearance of SEP-GluA1 (Kennedy et al., 2010). The exocytic events occurring adjacent to the PSD are mediated by syntaxin- 4 , which played a role in recycling endosome fusion to the spine plasma membrane. Disrupting syntaxin-4 blocks spine exocytosis and impairs LTP (Kennedy et al., 2010). The results of this study also suggest that different cargoes follow their own fate once they arrive at the spine surface. Although this study reported a requirement for syntaxin-4, but not for syntaxin-3 in LTP (Kennedy et al., 2010), other groups have demonstrated that LTP requires syntaxin-3, but not syntaxin-4 (Jurado et al., 2013; Arendt et al., 2015). Several explanations for these contradictory results have been extensively debated in the "Discussion" section of Jurado et al. (2013).

Recent work from Choquet and co-workers has provided a temporal profile of AMPAR trafficking for LTP expression by employing a novel approach that immobilizes surface AMPARs to prevent their diffusion on the cell surface (Penn et al., 2017). Biotin-tethered AMPAR subunit GluA1 or GluA2 can be expressed exogenously in cultured hippocampal neurons along with the endoplasmic-reticulum-retained biotin ligase (BirA-ER). In the presence of the biotinbinding protein NeutrAvidin, biotin-tethered AMPARs can be effectively crosslinked by NeutrAvidin, which reduces their surface diffusion as monitored by fluorescence recovery after photobleaching (FRAP; Penn et al., 2017). Using biotin-tethered GluA2 exogenously expressed in organotypic hippocampal slices prepared from GluA2-knockout mice, it was demonstrated that acute pre-treatment with NeutrAvidin to immobilize only pre-existing surface GluA2 results in a complete blockade of the short-term potentiation induced by a HFS LTP protocol. However, the hippocampal cells still express a detectable LTP, although small, indicating a contribution of exocytosis for LTP expression. Accordingly, the prevention of postsynaptic membrane fusion events by the intracellular application of tetanus toxin blocked HFS-induced LTP completely, but normal levels of short-term potentiation were still expressed, indicating a requirement of exocytosis for LTP expression (Penn et al., 2017).

It is very clear that LTP requires AMPAR exocytosis to sites adjacent to the PSD. These exocytosed receptors need to be relocated to synapses for synaptic potentiation (Makino and Malinow, 2009; Kennedy et al., 2010; Patterson et al., 2010). Interestingly, some populations of AMPARs may diffuse in and out between extrasynaptic and synaptic sites in order to tune synaptic transmission (Heine et al., 2008), and this receptor exchange organized on the surface membrane through lateral mobility is regulated dynamically by activity. Choquet and co-workers demonstrated that extrasynaptic surface AMPARs adjacent to the PSD arrive at synaptic sites through lateral diffusion upon LTP stimulation (Tardin et al., 2003; Opazo et al., 2010; Opazo and Choquet, 2011; Huganir and Nicoll, 2013; Chater and Goda, 2014; Constals et al., 2015; Compans et al., 2016; Penn et al., 2017). The lateral mobility of the surface GluA2 subunit inside nanodomains and/or outside of synapses was observed first using single-molecule fluorescence microscopy (Borgdorff and Choquet, 2002; Tardin et al., 2003). Lateral diffusion of surface GluA2 between extrasynaptic and synaptic sites is regulated by increased intracellular $\mathrm{Ca}^{2+}$, glutamate application, and glycine-induced stimulation, suggesting that the lateral diffusion of AMPARs may act as an important controlling step for synaptic plasticity (Borgdorff and Choquet, 2002; Tardin et al., 2003). Taken together, these reports suggest that a pre-existing surface pool of AMPARs are a prompt source to reach synapses via lateral diffusion and are then trapped at synapses for short-term potentiation, while newly exocytosed AMPARs from the recycling endosome upon LTP stimulation are the major source for sustaining LTP expression (Figure 1). Future work should address the intriguing possibility that the prompt reservoir of AMPARs for LTP is an extra-nanodomain or a true extrasynaptic region.

\section{STABILIZATION OF AMPARS AT SYNAPTIC SITES FOR LTP}

Laterally diffusing surface AMPARs must be trapped and immobilized at nanodomains on synaptic sites for LTP stabilization. Stargazin, an AMPAR auxiliary protein (Tomita et al., 2005; Hafner et al., 2015), was reported as a key molecule involved in the trapping and stabilization of AMPARs at synaptic sites during LTP (Opazo et al., 2010). LTP-inducing stimulation to activate NMDARs resulting in $\mathrm{Ca}^{2+}$ influx triggers CaMKII activation. CaMKII-mediated phosphorylation of the C-terminal PDZ-binding domain in Stargazin creates a highly negatively charged C-terminal tail of Stargazin so that it repulses the negatively charged membrane lipid. The C-terminal tail of Stargazin then unfolds, which favors its binding to PSD-95, thereby increasing the synaptic trapping of AMPARs on the nanodomains (Figure 1; Opazo et al., 2010; Opazo and Choquet, 2011; Choquet and Triller, 2013).

Besides these intracellular mechanisms of AMPAR trapping and stabilization at synaptic sites, LTP stabilization might also involve trans-synaptic mechanisms involving the extracellular 


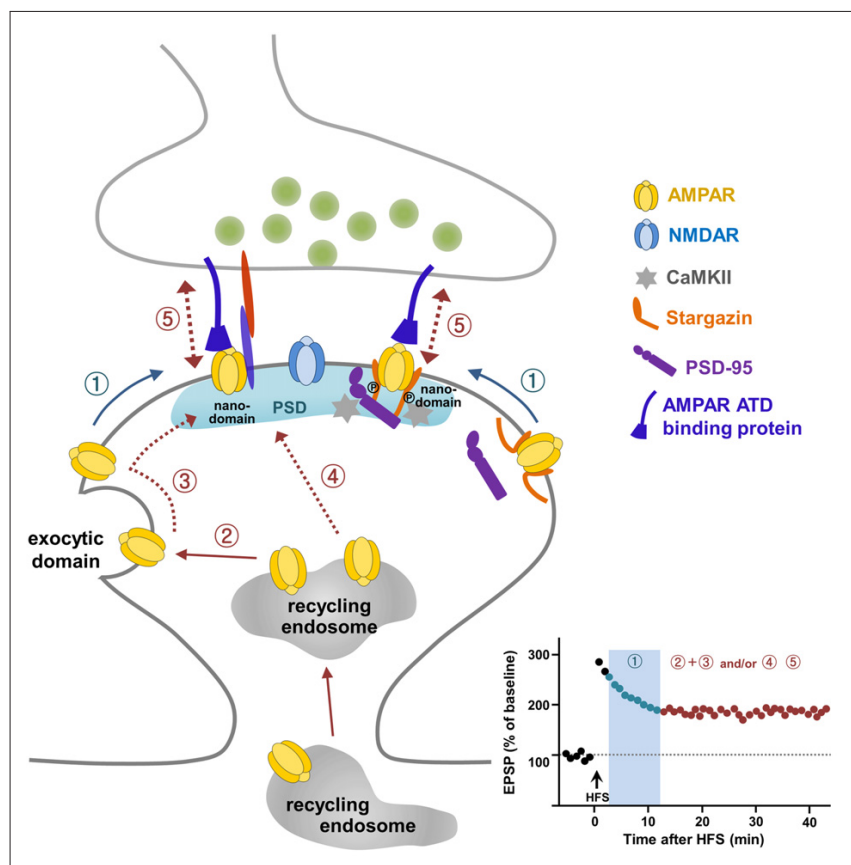

FIGURE 1 | A current model for $\alpha$-amino-3-hydroxy-5-methyl-4isoxazolepropionate (AMPA) receptor (AMPAR) trafficking during long-term potentiation (LTP). Pre-existing extrasynaptic surface AMPARs are mobilized to the synaptic sites upon LTP stimulation via lateral diffusion (1) and are trapped in nanodomains within the synapse, which is required for short-term potentiation (1) in LTP graph). AMPARs exocytosed from intracellular recycling endosomes to the exocytic domain adjacent to the postsynaptic density (PSD) replenish the extrasynaptic surface pool of AMPARs (2)), and these exocytosed AMPARs are mobilized laterally to reach the synaptic site (3) and supply AMPARs for maintaining LTP expression. The direct delivery of a portion of AMPARs on recycling endosomes to the synaptic site during LTP (4) cannot be ruled out. AMPARs trapped at the synapse are immobilized through the regulation of Stargazin and probably through the subunit-specific regulation of the AMPAR amino-terminal domain (ATD) interacting protein (5).

ATD of AMPARs. Indeed, the involvement of extracellular domains of AMPARs in LTP has recently been reported (DiazAlonso et al., 2017; Watson et al., 2017). The extracellular ATDs of GluA1 and GluA2 exert a subunit-specific role in synaptic trafficking of AMPARs. The ATD of GluA1, but not GluA2, is required for surface GluA1 translocation to synapses (Diaz-Alonso et al., 2017). GluA1 without ATD exhibits increased mobility in synapses and failed to sustain LTP (Diaz-Alonso et al., 2017), indicating a requirement for ATD of GluA1 in LTP stabilization. Further, a concept of a trans-synaptic molecular nanocolumn, stretching from the presynaptic neurotransmitter release site to the postsynaptic receptor cluster, has been introduced (Savtchenko and Rusakov, 2014; Tang et al., 2016; Biederer et al., 2017). LTD-triggering stimuli reorganized nanocolumns through trans-synaptic nanocluster realignment whereas LTP-triggering stimuli reorganized only postsynaptic nanoclusters with no changes in presynaptic nanoclusters (Tang et al., 2016). It might be possible to observe presynaptic nanocluster reorganization if LTP could be stabilized, which can be mediated by trans-synaptic communications.
Super-resolution imaging technologies (Hell and Wichmann, 1994; Betzig, 1995; Betzig et al., 2006; Rust et al., 2006; Manley et al., 2008) with 10- to 100-nm spatial resolution, such as stimulated emission depletion microscopy (STED), photoactivated localization microscopy (PALM), universal point accumulation in nanoscale topography (u-PAINT), direct stochastic optical reconstruction microscopy (dSTORM) and electron microscopy have demonstrated AMPAR nanodomains inside synapses, with $1-3$ of $80 \mathrm{~nm}$ clusters at each synapse and 20-25 AMPARs in each cluster (Nair et al., 2013), and trans-synaptic nanocolumns (Tang et al., 2016). Improvements in super-resolution imaging techniques and protein sensor development should allow greater manipulation and observation (Chater and Goda, 2014; Granger and Nicoll, 2014; Martineau et al., 2017). These improved methods will facilitate investigations as to whether new nanodomains, where AMPARs are trapped during LTP, and/or nanocoulmns are formed to mediate synaptic potentiation during LTP and whether AMPARs are trapped on pre-existing and/or newly formed nanodomains and/or nanocolumn, if generated, during LTP (Compans et al., 2016).

\section{SUMMARY AND PERSPECTIVES}

Over the past two decades, many laboratories have committed intensive effort to uncover mechanisms underlying AMPAR trafficking during LTP. These efforts, employing novel and advanced methods in electrophysiology, molecular and cellular biology, biochemistry, imaging and genetics provide a working model for how reserve pools of AMPARs are delivered to synapses for LTP (Figure 1). According to this model, pre-existing surface AMPARs are the first requirement for LTP expression. These pre-existing surface AMPARs are mobilized quickly to synaptic sites via lateral diffusion upon LTP stimulation and are trapped in microdomains within synapses (Figure 11). To sustain the expression of LTP, more AMPARs need to be delivered to the synaptic sites. This occurs via exocytosis of AMPARs from the recycling endosome to extrasynaptic sites to replenish the surface pool of AMPARs (Figure 1(2), which then diffuse laterally and are trapped at the synapses (Figure 1(3). In addition, it cannot be ruled out that a portion of AMPARs are supplied to the synaptic sites via exocytosis directly from the recycling endosomes (Figure 1(4)). Although the study by Penn et al. (2017) has advanced our understanding of LTP expression, some questions still remain to be addressed. Future development of molecular and opto-genetic manipulations and imaging technologies with greater spatial and temporal resoultion, will help determine whether subunits of AMPARs interplay with each other to contribute differentially to LTP expression. In addition, it will be of interest to investigate the relationship between AMPAR surface mobility and LTD, and, further, to elucidate whether different learning paradigms such as fear conditioning, water maze, passive avoidance, or novel object recognition utilize specific AMPAR trafficking mechanisms. Indeed, the C-terminal tails of GluA1 and GluA2 have been reported to exert differential roles in spatial learning and memory 
and contextual fear memory, respectively, suggesting a specific regulation of behavioral plasticity by AMPARs (Zhou et al., 2018).

The requirement of the C-terminal tail of GluA1 for LTP has been well accepted in the field since the sophisticated electrophysiology study of Shi et al. (2001). This work has resulted in many follow-up studies, whose main focus has been uncovering the nature of the molecules interacting with the C-terminal tails of AMPARs for the regulation of synaptic transmission and plasticity. However, the requirement of the C-terminal tail of GluA1 for LTP has been challenged (Granger et al., 2013), and subsequent studies have turned their attention toward the involvement of extracellular domains of AMPARs in LTP (Diaz-Alonso et al., 2017; Watson et al., 2017). Mobilization of surface AMPARs from extrasynaptic to synaptic sites is a well-recognized process for LTP expression. Stargazin has been identified as a regulator for LTP-triggered CaMKII-mediated trapping and immobilization of AMPARs diffusing in the membrane (Opazo et al., 2010). Analogous to the intracellular mechanism underlying immobilization of AMPARs at synapses by Stargazin, and given that studies highlight the importance of extracellular ATDs of AMPARs in LTP (Diaz-Alonso et al., 2017; Watson et al., 2017) and the reorganization of trans-synaptic nanocolumns by NMDAR activation (Tang et al., 2016), it is possible that trans-synaptic anchoring mechanisms, probably involving synaptic adhesion molecules, stabilize AMPARs by trapping them through their ATDs and preventing them from diffusing during LTP (Figure 15). Some synaptic adhesion molecules may be good candidates for anchoring through direct or indirect interactions with the ATD of AMPARs in the cleft space (Shipman and Nicoll, 2012; Aoto et al., 2013; Anderson et al., 2015; Jang et al., 2016; Gulisano et al., 2017; Varbanov and Dityatev, 2017; Bhouri et al., 2018).

\section{REFERENCES}

Abbas, A. K., Dozmorov, M., Li, R., Huang, F. S., Hellberg, F., Danielson, J., et al. (2009). Persistent LTP without triggered protein synthesis. Neurosci. Res. 63, 59-65. doi: 10.1016/j.neures.2008.10.008

Ahmad, M., Polepalli, J. S., Goswami, D., Yang, X., Kaeser-Woo, Y. J., Sudhof, T. C., et al. (2012). Postsynaptic complexin controls AMPA receptor exocytosis during LTP. Neuron 73, 260-267. doi: 10.1016/j.neuron.2011. 11.020 .

Anderson, G. R., Aoto, J., Tabuchi, K., Foldy, C., Covy, J., Yee, A. X., et al. (2015). $\beta$-neurexins control neural circuits by regulating synaptic endocannabinoid signaling. Cell 162, 593-606. doi: 10.1016/j.cell.2015.06.056

Anggono, V., and Huganir, R. L. (2012). Regulation of AMPA receptor trafficking and synaptic plasticity. Curr. Opin. Neurobiol. 22, 461-469. doi: 10.1016/j.conb. 2011.12.006

Aoto, J., Martinelli, D. C., Malenka, R. C., Tabuchi, K., and Sudhof, T. C. (2013). Presynaptic neurexin-3 alternative splicing trans-synaptically controls postsynaptic AMPA receptor trafficking. Cell 154, 75-88. doi: 10.1016/j.cell. 2013.05.060

Araki, Y., Lin, D. T., and Huganir, R. L. (2010). Plasma membrane insertion of the AMPA receptor GluA2 subunit is regulated by NSF binding and Q/R editing of the ion pore. Proc. Natl. Acad. Sci. U S A 107, 11080-11085. doi: 10.1073/pnas. 1006584107

Arendt, K. L., Zhang, Y., Jurado, S., Malenka, R. C., Sudhof, T. C., and Chen, L. (2015). Retinoic acid and LTP recruit postsynaptic AMPA receptors using distinct SNARE-dependent mechanisms. Neuron 86, 442-456. doi: 10.1016/j. neuron.2015.03.009
Further studies of extracellular trans-synaptic ATD regulation will add more information about the specific mechanisms by identifying the molecules involved in AMPAR trafficking during LTP. The recent visualization and measurement of intracellular transport of newly synthesized AMPARs during LTP (Hangen et al., 2018) provides a possible way to investigate the intracellular dynamics and mechanisms of synaptic key molecules that link the transition from the early to late phase of LTP, which might be contributed by local protein synthesis in dendrites (Sutton and Schuman, 2005; Sutton et al., 2006). Together, these approaches will further expand our understanding of LTP and open a new era in studies into how LTP affects synaptic plasticity and ultimately learning and memory.

\section{AUTHOR CONTRIBUTIONS}

The author confirms being the sole contributor of this work and approved it for publication.

\section{FUNDING}

The work in the Park laboratory was supported by the Original Technology Research Program for Brain Science of the National Research Foundation of Korea (NRF) funded by the Korean government (MSIT; no. 2018M3C7A1021848) and Korea Institute of Science and Technology (KIST) Institutional Program (2E27850).

\section{ACKNOWLEDGMENTS}

The author thanks E. Cho for valuable comments on the manuscript.

Bagal, A. A., Kao, J. P., Tang, C.-M., and Thompson, S. M. (2005). Long-term potentiation of exogenous glutamate responses at single dendritic spines. Proc. Natl. Acad. Sci. U S A 102, 14434-14439. doi: 10.1073/pnas.05019 56102

Barria, A., Muller, D., Derkach, V., Griffith, L. C., and Soderling, T. R. (1997). Regulatory phosphorylation of AMPA-type glutamate receptors by CaM-KII during long-term potentiation. Science 276, 2042-2045. doi: 10.1126/science. 276.5321.2042

Bear, M. F., and Kirkwood, A. (1993). Neocortical long-term potentiation. Curr. Opin. Neurobiol. 3, 197-202. doi: 10.1016/0959-4388(93)90210-p

Benke, T. A., Luthi, A., Isaac, J. T., and Collingridge, G. L. (1998). Modulation of AMPA receptor unitary conductance by synaptic activity. Nature 393, 793-797. doi: $10.1038 / 31709$

Benke, T., and Traynelis, S. F. (2018). AMPA-type glutamate receptor conductance changes and plasticity: still a lot of noise. Neurochem. Res. doi: 10.1007/s11064018-2491-1 [Epub ahead of print].

Betzig, E. (1995). Proposed method for molecular optical imaging. Opt. Lett. 20, 237-239. doi: 10.1364/ol.20.000237

Betzig, E., Patterson, G. H., Sougrat, R., Lindwasser, O. W., Olenych, S., Bonifacino, J. S., et al. (2006). Imaging intracellular fluorescent proteins at nanometer resolution. Science 313, 1642-1645. doi: 10.1126/science. 1127344

Bhouri, M., Morishita, W., Temkin, P., Goswami, D., Kawabe, H., Brose, N., et al. (2018). Deletion of LRRTM1 and LRRTM2 in adult mice impairs basal AMPA receptor transmission and LTP in hippocampal CA1 pyramidal neurons. Proc. Natl. Acad. Sci. U S A 115, E5382-E5389. doi: 10.1073/pnas.1803 280115 
Biederer, T., Kaeser, P. S., and Blanpied, T. A. (2017). Transcellular nanoalignment of synaptic function. Neuron 96, 680-696. doi: 10.1016/j.neuron.2017.10.006

Bliss, T. V., and Lømo, T. (1973). Long-lasting potentiation of synaptic transmission in the dentate area of the anaesthetized rabbit following stimulation of the perforant path. J. Physiol. 232, 331-356. doi: 10.1113/jphysiol.1973.sp010273

Boehm, J., Kang, M.-G., Johnson, R. C., Esteban, J., Huganir, R. L., and Malinow, R. (2006). Synaptic incorporation of AMPA receptors during LTP is controlled by a PKC phosphorylation site on GluR1. Neuron 51, 213-225. doi: 10.1016/j. neuron.2006.06.013

Borgdorff, A. J., and Choquet, D. (2002). Regulation of AMPA receptor lateral movements. Nature 417, 649-653. doi: 10.1038/nature00780

Bredt, D. S., and Nicoll, R. A. (2003). AMPA receptor trafficking at excitatory synapses. Neuron 40, 361-379. doi: 10.1016/s0896-6273(03)00640-8

Bryant, N. J., Govers, R., and James, D. E. (2002). Regulated transport of the glucose transporter GLUT4. Nat. Rev. Mol. Cell Biol. 3, 267-277. doi: $10.1038 / \mathrm{nrm} 782$

Chater, T. E., and Goda, Y. (2014). The role of AMPA receptors in postsynaptic mechanisms of synaptic plasticity. Front. Cell. Neurosci. 8:401. doi: 10.3389/fncel.2014.00401

Cho, E., Kim, D.-H., Hur, Y.-N., Whitcomb, D. J., Regan, P., Hong, J.-H., et al. (2015). Cyclin Y inhibits plasticity-induced AMPA receptor exocytosis and LTP. Sci. Rep. 5:12624. doi: 10.1038/srep12624

Choquet, D., and Triller, A. (2013). The dynamic synapse. Neuron 80, 691-703. doi: 10.1016/j.neuron.2013.10.013

Compans, B., Choquet, D., and Hosy, E. (2016). Review on the role of AMPA receptor nano-organization and dynamic in the properties of synaptic transmission. Neurophotonics 3:041811. doi: 10.1117/1.nph.3.4.041811

Constals, A., Penn, A. C., Compans, B., Toulme, E., Phillipat, A., Marais, S., et al. (2015). Glutamate-induced AMPA receptor desensitization increases their mobility and modulates short-term plasticity through unbinding from Stargazin. Neuron 85, 787-803. doi: 10.1016/j.neuron.2015.01.012

Cooney, J. R., Hurlburt, J. L., Selig, D. K., Harris, K. M., and Fiala, J. C. (2002). Endosomal compartments serve multiple hippocampal dendritic spines from a widespread rather than a local store of recycling membrane. J. Neurosci. 22, 2215-2224. doi: 10.1523/jneurosci.22-06-02215.2002

Correia, S. S., Bassani, S., Brown, T. C., Lise, M. F., Backos, D. S., El-Husseini, A., et al. (2008). Motor protein-dependent transport of AMPA receptors into spines during long-term potentiation. Nat. Neurosci. 11, 457-466. doi: 10.1038/nn2063

Diaz-Alonso, J., Sun, Y. J., Granger, A. J., Levy, J. M., Blankenship, S. M., and Nicoll, R. A. (2017). Subunit-specific role for the amino-terminal domain of AMPA receptors in synaptic targeting. Proc. Natl. Acad. Sci. U S A 114, 7136-7141. doi: 10.1073/pnas.1707472114

Dingledine, R., Borges, K., Bowie, D., and Traynelis, S. F. (1999). The glutamate receptor ion channels. Pharmacol. Rev. 51, 7-61.

Durand, G. M., Kovalchuk, Y., and Konnerth, A. (1996). Long-term potentiation and functional synapse induction in developing hippocampus. Nature 381, 71-75. doi: 10.1038/381071a0

Ehlers, M. D. (2000). Reinsertion or degradation of AMPA receptors determined by activity-dependent endocytic sorting. Neuron 28, 511-525. doi: 10.1016/s0896-6273(00)00129-x

Ehlers, M. D. (2013). Dendritic trafficking for neuronal growth and plasticity. Biochem. Soc. Trans. 41, 1365-1382. doi: 10.1042/bst20130081

Emptage, N., Bliss, T. V., and Fine, A. (1999). Single synaptic events evoke NMDA receptor-mediated release of calcium from internal stores in hippocampal dendritic spines. Neuron 22, 115-124. doi: 10.1016/s0896-6273(00) 80683-2

Emptage, N. J., Reid, C. A., Fine, A., and Bliss, T. V. (2003). Optical quantal analysis reveals a presynaptic component of LTP at hippocampal Schaffer-associational synapses. Neuron 38, 797-804. doi: 10.1016/s0896-6273(03)00325-8

Enoki, R., Hu, Y. L., Hamilton, D., and Fine, A. (2009). Expression of long-term plasticity at individual synapses in hippocampus is graded, bidirectional and mainly presynaptic: optical quantal analysis. Neuron 62, 242-253. doi: 10.1016/j.neuron.2009.02.026

Esteban, J. A., Shi, S. H., Wilson, C., Nuriya, M., Huganir, R. L., and Malinow, R. (2003). PKA phosphorylation of AMPA receptor subunits controls synaptic trafficking underlying plasticity. Nat. Neurosci. 6, 136-143. doi: 10.1038/nn997
Folci, A., Murru, L., Vezzoli, E., Ponzoni, L., Gerosa, L., Moretto, E., et al. (2016). Myosin IXa binds AMPAR and regulates synaptic structure, LTP, and cognitive function. Front. Mol. Neurosci. 9:1. doi: 10.3389/fnmol.2016.00001

Gomperts, S. N., Rao, A., Craig, A. M., Malenka, R. C., and Nicoll, R. A. (1998). Postsynaptically silent synapses in single neuron cultures. Neuron 21, 1443-1451. doi: 10.1016/s0896-6273(00)80662-5

Govers, R., Coster, A. C., and James, D. E. (2004). Insulin increases cell surface GLUT4 levels by dose dependently discharging GLUT4 into a cell surface recycling pathway. Mol Cell Biol 24, 6456-6466. doi: 10.1128/mcb.24.14.64566466.2004

Granger, A. J., and Nicoll, R. A. (2014). Expression mechanisms underlying long-term potentiation: a postsynaptic view, 10 years on. Philos. Trans. R. Soc. Lond. B Biol. Sci. 369:20130136. doi: 10.1098/rstb.2013.0136.

Granger, A. J., Shi, Y., Lu, W., Cerpas, M., and Nicoll, R. A. (2013). LTP requires a reserve pool of glutamate receptors independent of subunit type. Nature 493, 495-500. doi: 10.1038/nature11775

Grant, B., Zhang, Y., Paupard, M. C., Lin, S. X., Hall, D. H., and Hirsh, D. (2001). Evidence that RME-1, a conserved C. elegans EH-domain protein, functions in endocytic recycling. Nat. Cell Biol. 3, 573-579. doi: 10.1038/35078549

Guilherme, A., Soriano, N. A., Furcinitti, P. S., and Czech, M. P. (2004), Role of EHD1 and EHBP1 in perinuclear sorting and insulin-regulated GLUT4 recycling in 3T3-L1 adipocytes. J. Biol. Chem. 279, 40062-40075. doi: $10.1074 /$ jbc.M401918200

Gulisano, W., Bizzoca, A., Gennarini, G., Palmeri, A., and Puzzo, D. (2017). Role of the adhesion molecule F3/Contactin in synaptic plasticity and memory. Mol. Cell. Neurosci. 81, 64-71. doi: 10.1016/j.mcn.2016.12.003

Hafner, A. S., Penn, A. C., Grillo-Bosch, D., Retailleau, N., Poujol, C., Philippat, A., et al. (2015). Lengthening of the stargazin cytoplasmic tail increases synaptic transmission by promoting interaction to deeper domains of PSD-95. Neuron 86, 475-489. doi: 10.1016/j.neuron.2015.03.013

Hangen, E., Cordelieres, F. P., Petersen, J. D., Choquet, D., and Coussen, F. (2018). Neuronal activity and intracellular calcium levels regulate intracellular transport of newly synthesized AMPAR. Cell Rep. 24, 1001.e3-1012.e3. doi: 10.1016/j.celrep.2018.06.095

Harvey, C. D., and Svoboda, K. (2007). Locally dynamic synaptic learning rules in pyramidal neuron dendrites. Nature 450, 1195-1200. doi: 10.1038/nature 06416

Hayashi, Y., Shi, S. H., Esteban, J. A., Piccini, A., Poncer, J. C., and Malinow, R. (2000). Driving AMPA receptors into synapses by LTP and CaMKII: requirement for GluR1 and PDZ domain interaction. Science 287, 2262-2267. doi: $10.1126 /$ science.287.5461.2262

Hebb, D. O. (1949). The Organization of Behavior. New York, NY: Wiley \& Sons.

Heine, M., Thoumine, O., Mondin, M., Tessier, B., Giannone, G., and Choquet, D. (2008). Activity-independent and subunit-specific recruitment of functional AMPA receptors at neurexin/neuroligin contacts. Proc. Natl. Acad. Sci. U S A 105, 20947-20952. doi: 10.1073/pnas.0804007106

Hell, S. W., and Wichmann, J. (1994). Breaking the diffraction resolution limit by stimulated emission: stimulated-emission-depletion fluorescence microscopy. Opt. Lett. 19, 780-782. doi: 10.1364/ol.19.000780

Heynen, A. J., Quinlan, E. M., Bae, D. C., and Bear, M. F. (2000). Bidirectional, activity-dependent regulation of glutamate receptors in the adult hippocampus in vivo. Neuron 28, 527-536. doi: 10.1016/s0896-6273(00)00130-6

Hollmann, M., and Heinemann, S. (1994). Cloned glutamate receptors. Annu. Rev. Neurosci. 17, 31-108. doi: 10.1146/annurev.ne.17.030194.000335

Huganir, R. L., and Nicoll, R. A. (2013). AMPARs and synaptic plasticity: the last 25 years. Neuron 80, 704-717. doi: 10.1016/j.neuron.2013.10.025

Isaac, J. T., Nicoll, R. A., and Malenka, R. C. (1995). Evidence for silent synapses: implications for the expression of LTP. Neuron 15, 427-434. doi: 10.1016/08966273(95)90046-2

Isaac, J. T., Oliet, S. H., Hjelmstad, G. O., Nicoll, R. A., and Malenka, R. C. (1996). Expression mechanisms of long-term potentiation in the hippocampus. J. Physiol. Paris 90, 299-303. doi: 10.1016/s0928-4257(97)87901-6

Jang, S., Oh, D., Lee, Y., Hosy, E., Shin, H., Van Riesen, C., et al. (2016). Synaptic adhesion molecule IgSF11 regulates synaptic transmission and plasticity. Nat. Neurosci. 19, 84-93. doi: 10.1038/nn.4176

Jaskolski, F., and Henley, J. M. (2009). Synaptic receptor trafficking: the lateral point of view. Neuroscience 158, 19-24. doi: 10.1016/j.neuroscience.2008. 01.075 
Jia, Z., Agopyan, N., Miu, P., Xiong, Z., Henderson, J., Gerlai, R., et al. (1996). Enhanced LTP in mice deficient in the AMPA receptor GluR2. Neuron 17, 945-956. doi: 10.1016/s0896-6273(00)80225-1

Johnstone, V. P., and Raymond, C. R. (2011). A protein synthesis and nitric oxide-dependent presynaptic enhancement in persistent forms of long-term potentiation. Learn. Mem. 18, 625-633. doi: 10.1101/lm.2245911

Jurado, S., Goswami, D., Zhang, Y., Molina, A. J., Südhof, T. C., and Malenka, R. C. (2013). LTP requires a unique postsynaptic SNARE fusion machinery. Neuron 77, 542-558. doi: 10.1016/j.neuron.2012.11.029

Kennedy, M. J., Davison, I. G., Robinson, C. G., and Ehlers, M. D. (2010). Syntaxin4 defines a domain for activity-dependent exocytosis in dendritic spines. Cell 141, 524-535. doi: 10.1016/j.cell.2010.02.042

Kerchner, G. A., and Nicoll, R. A. (2008). Silent synapses and the emergence of a postsynaptic mechanism for LTP. Nat. Rev. Neurosci. 9, 813-825. doi: $10.1038 / \mathrm{nrn} 2501$

Kessels, H. W., and Malinow, R. (2009). Synaptic AMPA receptor plasticity and behavior. Neuron 61, 340-350. doi: 10.1038/nrn2501

Kirkwood, A., Dudek, S. M., Gold, J. T., Aizenman, C. D., and Bear, M. F. (1993). Common forms of synaptic plasticity in the hippocampus and neocortex in vitro. Science 260, 1518-1521. doi: 10.1126/science.8502997

Kopec, C. D., Li, B., Wei, W., Boehm, J., and Malinow, R. (2006). Glutamate receptor exocytosis and spine enlargement during chemically induced long-term potentiation. J. Neurosci. 26, 2000-2009. doi: 10.1523/JNEUROSCI. 3918-05.2006

Kopec, C. D., Kessels, H. W., Bush, D. E., Cain, C. K., LeDoux, J. E., and Malinow, R. (2007a). A robust automated method to analyze rodent motion during fear conditioning. Neuropharmacology 52, 228-233. doi: 10.1016/j. neuropharm.2006.07.028

Kopec, C. D., Real, E., Kessels, H. W., and Malinow, R. (2007b). GluR1 links structural and functional plasticity at excitatory synapses. J. Neurosci. 27, 13706-13718. doi: 10.1523/JNEUROSCI.3503-07.2007

Kullmann, D. M. (2012). The mother of all battles 20 years on: is LTP expressed pre- or postsynaptically? J. Physiol. 590, 2213-2216. doi: 10.1113/jphysiol.2011. 221127

Kullmann, D. M., and Siegelbaum, S. A. (1995). The site of expression of NMDA receptor-dependent LTP: new fuel for an old fire. Neuron 15, 997-1002. doi: 10.1016/0896-6273(95)90089-6

Lampson, M. A., Schmoranzer, J., Zeigerer, A., Simon, S. M., and McGraw, T. E. (2001). Insulin-regulated release from the endosomal recycling compartment is regulated by budding of specialized vesicles. Mol. Biol. Cell 12, 3489-3501. doi: 10.1091/mbc.12.11.3489

Lee, H.-K., Barbarosie, M., Kameyama, K., Bear, M. F., and Huganir, R. L. (2000). Regulation of distinct AMPA receptor phosphorylation sites during bidirectional synaptic plasticity. Nature 405, 955-959. doi: 10.1038/35016089

Lee, S. J., Escobedo-Lozoya, Y., Szatmari, E. M., and Yasuda, R. (2009). Activation of CaMKII in single dendritic spines during long-term potentiation. Nature 458, 299-304. doi: 10.1038/nature07842

Lee, H.-K., Takamiya, K., Han, J.-S., Man, H., Kim, C.-H., Rumbaugh, G., et al. (2003). Phosphorylation of the AMPA receptor GluR1 subunit is required for synaptic plasticity and retention of spatial memory. Cell 112, 631-643. doi: 10.1016/s0092-8674(03)00122-3

Liao, D., Hessler, N. A., and Malinow, R. (1995). Activation of postsynaptically silent synapses during pairing-induced LTP in CA1 region of hippocampal slice. Nature 375, 400-404. doi: 10.1038/375400a0

Liao, D., Zhang, X., O’Brien, R., Ehlers, M. D., and Huganir, R. L. (1999). Regulation of morphological postsynaptic silent synapses in developing hippocampal neurons. Nat. Neurosci. 2, 37-43. doi: 10.1038/4540

Lin, S. X., Grant, B., Hirsh, D., and Maxfield, F. R. (2001). Rme-1 regulates the distribution and function of the endocytic recycling compartment in mammalian cells. Nat. Cell Biol. 3, 567-572. doi: 10.1038/35078543

Lin, D.-T., Makino, Y., Sharma, K., Hayashi, T., Neve, R., Takamiya, K., et al. (2009). Regulation of AMPA receptor extrasynaptic insertion by $4.1 \mathrm{~N}$, phosphorylation and palmitoylation. Nat. Neurosci. 12, 879-887. doi: $10.1038 / \mathrm{nn} .2351$

Lisman, J. (1994). The CaM kinase II hypothesis for the storage of synaptic memory. Trends Neurosci. 17, 406-412. doi: 10.1016/0166-2236(94)90014-0

Lisman, J., Malenka, R. C., Nicoll, R. A., and Malinow, R. (1997). Learning mechanisms: the case for CaM-KII. Science 276, 2001-2002. doi: $10.1126 /$ science.276.5321.2001
Lisman, J., Yasuda, R., and Raghavachari, S. (2012). Mechanisms of CaMKII action in long-term potentiation. Nat. Rev. Neurosci. 13, 169-182. doi: $10.1038 / \mathrm{nrn} 3192$

Lledo, P.-M., Zhang, X., Südhof, T. C., Malenka, R. C., and Nicoll, R. A. (1998). Postsynaptic membrane fusion and long-term potentiation. Science 279, 399-403. doi: 10.1126/science.279.5349.399

Lu, J., Helton, T. D., Blanpied, T. A., Rácz, B., Newpher, T. M., Weinberg, R. J., et al. (2007). Postsynaptic positioning of endocytic zones and AMPA receptor cycling by physical coupling of dynamin-3 to Homer. Neuron 55, 874-889. doi: 10.1016/j.neuron.2007.06.041

Lu, W.-Y., Man, H.-Y., Ju, W., Trimble, W. S., Macdonald, J. F., and Wang, Y. T. (2001). Activation of synaptic NMDA receptors induces membrane insertion of new AMPA receptors and LTP in cultured hippocampal neurons. Neuron 29, 243-254. doi: 10.1016/s0896-6273(01)00194-5

Lu, W., and Roche, K. W. (2012). Posttranslational regulation of AMPA receptor trafficking and function. Curr. Opin. Neurobiol. 22, 470-479. doi: 10.1016/j. conb.2011.09.008

Makino, H., and Malinow, R. (2009). AMPA receptor incorporation into synapses during LTP: the role of lateral movement and exocytosis. Neuron 64, 381-390 doi: 10.1016/j.neuron.2009.08.035

Malenka, R. C. (2003). The long-term potential of LTP. Nat. Rev. Neurosci. 4 923-926. doi: 10.1038/nrn1258

Malenka, R. C., Kauer, J. A., Perkel, D. J., Mauk, M. D., Kelly, P. T., Nicoll, R. A. et al. (1989). An essential role for postsynaptic calmodulin and protein kinase activity in long-term potentiation. Nature 340, 554-557. doi: 10.1038/ $340554 \mathrm{a} 0$

Malenka, R. C., and Nicoll, R. A. (1999). Long-term potentiation-a decade of progress? Science 285, 1870-1874. doi: 10.1126/science.285.5435.1870

Malinow, R., and Malenka, R. C. (2002). AMPA receptor trafficking and synaptic plasticity. Annu. Rev. Neurosci. 25, 103-126. doi: 10.1146/annurev.neuro.25. 112701.142758

Malinow, R., Schulman, H., and Tsien, R. W. (1989). Inhibition of postsynaptic PKC or CaMKII blocks induction but not expression of LTP. Science 245, 862-866. doi: 10.1126/science. 2549638

Manley, S., Gillette, J. M., Patterson, G. H., Shroff, H., Hess, H. F., Betzig, E., et al. (2008). High-density mapping of single-molecule trajectories with photoactivated localization microscopy. Nat. Methods 5, 155-157. doi: $10.1038 /$ nmeth. 1176

Martineau, M., Somasundaram, A., Grimm, J. B., Gruber, T. D., Choquet, D., Taraska, J. W., et al. (2017). Semisynthetic fluorescent $\mathrm{pH}$ sensors for imaging exocytosis and endocytosis. Nat. Commun. 8:1412. doi: 10.1038/s41467-01701752-5

Matsuzaki, M., Honkura, N., Ellis-Davies, G. C., and Kasai, H. (2004). Structural basis of long-term potentiation in single dendritic spines. Nature 429, 761-766. doi: $10.1038 /$ nature 02617

Maximov, A., Tang, J., Yang, X., Pang, Z. P., and Sudhof, T. C. (2009). Complexin controls the force transfer from SNARE complexes to membranes in fusion. Science 323, 516-521. doi: 10.1126/science.1166505

Miesenbock, G., De Angelis, D. A., and Rothman, J. E. (1998). Visualizing secretion and synaptic transmission with $\mathrm{pH}$-sensitive green fluorescent proteins. Nature 394, 192-195. doi: 10.1038/28190

Nair, D., Hosy, E., Petersen, J. D., Constals, A., Giannone, G., Choquet, D., et al. (2013). Super-resolution imaging reveals that AMPA receptors inside synapses are dynamically organized in nanodomains regulated by PSD95. J. Neurosci. 33, 13204-13224. doi: 10.1523/JNEUROSCI.2381-12.2013

Nicoll, R. A. (2003). Expression mechanisms underlying long-term potentiation: a postsynaptic view. Philos. Trans. R. Soc. Lond. B Biol. Sci. 358, 721-726 doi: $10.1098 /$ rstb.2002.1228

Nicoll, R. A., and Malenka, R. C. (1995). Contrasting properties of two forms of long-term potentiation in the hippocampus. Nature 377, 115-118. doi: $10.1038 / 377115 \mathrm{a} 0$

Nicoll, R. A., and Roche, K. W. (2013). Long-term potentiation: peeling the onion. Neuropharmacology 74, 18-22. doi: 10.1016/j.neuropharm.2013.02.010

Nicoll, R. A., and Schmitz, D. (2005). Synaptic plasticity at hippocampal mossy fibre synapses. Nat. Rev. Neurosci. 6, 863-876. doi: 10.1038/nrn1786

Nusser, Z., Lujan, R., Laube, G., Roberts, J. D., Molnar, E., and Somogyi, P. (1998). Cell type and pathway dependence of synaptic AMPA receptor number and variability in the hippocampus. Neuron 21, 545-559. doi: 10.1016/s08966273(00)80565-6 
Opazo, P., and Choquet, D. (2011). A three-step model for the synaptic recruitment of AMPA receptors. Mol. Cell. Neurosci. 46, 1-8. doi: 10.1016/j. mcn.2010.08.014

Opazo, P., Labrecque, S., Tigaret, C. M., Frouin, A., Wiseman, P. W., De Koninck, P., et al. (2010). CaMKII triggers the diffusional trapping of surface AMPARs through phosphorylation of stargazin. Neuron 67, 239-252. doi: 10.1016/j.neuron.2010.06.007

Padamsey, Z., and Emptage, N. (2014). Two sides to long-term potentiation: a view towards reconciliation. Philos. Trans. R. Soc. Lond. B Biol. Sci. 369:20130154. doi: $10.1098 /$ rstb.2013.0154

Park, M., Penick, E. C., Edwards, J. G., Kauer, J. A., and Ehlers, M. D. (2004). Recycling endosomes supply AMPA receptors for LTP. Science 305, 1972-1975. doi: 10.1126/science.1102026

Patterson, M. A., Szatmari, E. M., and Yasuda, R. (2010). AMPA receptors are exocytosed in stimulated spines and adjacent dendrites in a Ras-ERKdependent manner during long-term potentiation. Proc. Natl. Acad. Sci. US A 107, 15951-15956. doi: 10.1073/pnas.0913875107

Penn, A. C., Zhang, C. L., Georges, F., Royer, L., Breillat, C., Hosy, E., et al. (2017). Hippocampal LTP and contextual learning require surface diffusion of AMPA receptors. Nature 549, 384-388. doi: 10.1038/nature23658

Petralia, R. S., Esteban, J. A., Wang, Y.-X., Partridge, J. G., Zhao, H.-M., Wenthold, R. J., et al. (1999). Selective acquisition of AMPA receptors over postnatal development suggests a molecular basis for silent synapses. Nat. Neurosci. 2, 31-36. doi: 10.1038/4532

Petrini, E. M., Lu, J., Cognet, L., Lounis, B., Ehlers, M. D., and Choquet, D. (2009). Endocytic trafficking and recycling maintain a pool of mobile surface AMPA receptors required for synaptic potentiation. Neuron 63, 92-105. doi: 10.1016/j. neuron.2009.05.025

Prekeris, R., Klumperman, J., Chen, Y. A., and Scheller, R. H. (1998). Syntaxin 13 mediates cycling of plasma membrane proteins via tubulovesicular recycling endosomes. J. Cell Biol. 143, 957-971. doi: 10.1083/jcb.143. 4.957

Reim, K., Mansour, M., Varoqueaux, F., Mcmahon, H. T., Sudhof, T. C., Brose, N., et al. (2001). Complexins regulate a late step in $\mathrm{Ca}^{2+}$-dependent neurotransmitter release. Cell 104, 71-81. doi: 10.1016/s0092-8674(01) 00192-1

Reymann, K. G., and Frey, J. U. (2007). The late maintenance of hippocampal LTP: requirements, phases, 'synaptic tagging', 'late-associativity' and implications. Neuropharmacology 52, 24-40. doi: 10.1016/j.neuropharm.2006. 07.026

Rust, M. J., Bates, M., and Zhuang, X. (2006). Sub-diffraction-limit imaging by stochastic optical reconstruction microscopy (STORM). Nat. Methods 3, 793-795. doi: 10.1038/nmeth929

Savtchenko, L. P., and Rusakov, D. A. (2014). Moderate AMPA receptor clustering on the nanoscale can efficiently potentiate synaptic current. Philos. Trans. R. Soc. Lond. B Biol. Sci. 369:20130167. doi: 10.1098/rstb.2013.0167

Schnell, E., and Nicoll, R. A. (2001). Hippocampal synaptic transmission and plasticity are preserved in myosin Va mutant mice. J. Neurophysiol. 85, 1498-1501. doi: 10.1152/jn.2001.85.4.1498

Schuman, E. M., Dynes, J. L., and Steward, O. (2006). Synaptic regulation of translation of dendritic mRNAs. J. Neurosci. 26, 7143-7146. doi: 10.1523/JNEUROSCI.1796-06.2006

Shi, S., Hayashi, Y., Esteban, J. A., and Malinow, R. (2001). Subunit-specific rules governing AMPA receptor trafficking to synapses in hippocampal pyramidal neurons. Cell 105, 331-343. doi: 10.1016/S0092-8674(01) 00321-X

Shi, S.-H., Hayashi, Y., Petralia, R. S., Zaman, S. H., Wenthold, R. J., Svoboda, K., et al. (1999). Rapid spine delivery and redistribution of AMPA receptors after synaptic NMDA receptor activation. Science 284, 1811-1816. doi: $10.1126 /$ science.284.5421.1811

Shipman, S. L., and Nicoll, R. A. (2012). A subtype-specific function for the extracellular domain of neuroligin 1 in hippocampal LTP. Neuron 76, 309-316. doi: 10.1016/j.neuron.2012.07.024

Silva, A. J., Stevens, C. F., Tonegawa, S., and Wang, Y. (1992). Deficient hippocampal long-term potentiation in $\alpha$-calcium-calmodulin kinase II mutant mice. Science 257, 201-206. doi: 10.1126/science.1378648

Song, I., and Huganir, R. L. (2002). Regulation of AMPA receptors during synaptic plasticity. Trends Neurosci. 25, 578-588. doi: 10.1016/s0166-2236(02) 02270-1
Sutton, M. A., and Schuman, E. M. (2005). Local translational control in dendrites and its role in long-term synaptic plasticity. J. Neurobiol. 64, 116-131. doi: 10.1002/neu.20152

Sutton, M. A., Ito, H. T., Cressy, P., Kempf, C., Woo, J. C., and Schuman, E. M. (2006). Miniature neurotransmission stabilizes synaptic function via tonic suppression of local dendritic protein synthesis. Cell 125, 785-799. doi: 10.1016/j.cell.2006.03.040

Takumi, Y., Ramirez-Leon, V., Laake, P., Rinvik, E., and Ottersen, O. P. (1999). Different modes of expression of AMPA and NMDA receptors in hippocampal synapses. Nat. Neurosci. 2, 618-624. doi: 10.1038/10172

Tang, A.-H., Chen, H., Li, T. P., Metzbower, S. R., Macgillavry, H. D., and Blanpied, T. A. (2016). A trans-synaptic nanocolumn aligns neurotransmitter release to receptors. Nature 536, 210-214. doi: 10.1038/nature 19058

Tardin, C., Cognet, L., Bats, C., Lounis, B., and Choquet, D. (2003). Direct imaging of lateral movements of AMPA receptors inside synapses. EMBO J. 22, 4656-4665. doi: 10.1093/emboj/cdg463

Temkin, P., Morishita, W., Goswami, D., Arendt, K., Chen, L., and Malenka, R. (2017). The retromer supports AMPA receptor trafficking during LTP. Neuron 94, 74.e5-82.e5. doi: 10.1016/j.neuron.2017.03.020

Tomita, S., Adesnik, H., Sekiguchi, M., Zhang, W., Wada, K., Howe, J. R., et al. (2005). Stargazin modulates AMPA receptor gating and trafficking by distinct domains. Nature 435, 1052-1058. doi: 10.1038/nature 03624

Traynelis, S. F., Wollmuth, L. P., Mcbain, C. J., Menniti, F. S., Vance, K. M., Ogden, K. K., et al. (2010). Glutamate receptor ion channels: structure, regulation and function. Pharmacol. Rev. 62, 405-496. doi: 10.1124/pr.109. 002451

Ullrich, O., Reinsch, S., Urbé, S., Zerial, M., and Parton, R. G. (1996) Rab11 regulates recycling through the pericentriolar recycling endosome. J. Cell Biol. 135, 913-924. doi: 10.1083/jcb.135.4.913

Varbanov, H., and Dityatev, A. (2017). Regulation of extrasynaptic signaling by polysialylated NCAM: impact for synaptic plasticity and cognitive functions. Mol. Cell. Neurosci. 81, 12-21. doi: 10.1016/j.mcn.2016.11.005

Villers, A., Godaux, E., and Ris, L. (2012). Long-lasting LTP requires neither repeated trains for its induction nor protein synthesis for its development. PLoS One 7:e40823. doi: 10.1371/journal.pone.0040823

Wang, Z., Edwards, J. G., Riley, N., Provance, D. W. Jr., Karcher, R., Li, X. D., et al. (2008). Myosin Vb mobilizes recycling endosomes and AMPA receptors for postsynaptic plasticity. Cell 135, 535-548. doi: 10.1016/j.cell.2008. 09.057

Ward, B., Mcguinness, L., Akerman, C. J., Fine, A., Bliss, T. V., and Emptage, N. J. (2006). State-dependent mechanisms of LTP expression revealed by optical quantal analysis. Neuron 52, 649-661. doi: 10.1016/j.neuron.2006.10.007

Watson, J. F., Ho, H., and Greger, I. H. (2017). Synaptic transmission and plasticity require AMPA receptor anchoring via its N-terminal domain. Elife 6:e23024 doi: 10.7554/eLife. 23024

Wenthold, R. J., Petralia, R. S., Blahos, J. II., and Niedzielski, A. S. (1996). Evidence for multiple AMPA receptor complexes in hippocampal CA1/CA2 neurons. J. Neurosci. 16, 1982-1989. doi: 10.1523/jneurosci.16-06-01982.1996

Whitlock, J. R., Heynen, A. J., Shuler, M. G., and Bear, M. F. (2006). Learning induces long-term potentiation in the hippocampus. Science 313, 1093-1097. doi: $10.1126 /$ science. 1128134

Wisden, W., and Seeburg, P. H. (1993). Mammalian ionotropic glutamate receptors. Curr. Opin. Neurobiol. 3, 291-298. doi: 10.1016/0959 4388(93)90120-N

Wu, D., Bacaj, T., Morishita, W., Goswami, D., Arendt, K. L., Xu, W., et al. (2017) Postsynaptic synaptotagmins mediate AMPA receptor exocytosis during LTP. Nature 544, 316-321. doi: 10.1038/nature21720

Yudowski, G. A., Puthenveedu, M. A., Leonoudakis, D., Panicker, S., Thorn, K. S., Beattie, E. C., et al. (2007). Real-time imaging of discrete exocytic events mediating surface delivery of AMPA receptors. J. Neurosci. 27, 11112-11121. doi: 10.1523/JNEUROSCI.2465-07.2007

Zamanillo, D., Sprengel, R., Hvalby, O., Jensen, V., Burnashev, N., Rozov, A., et al. (1999). Importance of AMPA receptors for hippocampal synaptic plasticity but not for spatial learning. Science 284, 1805-1811. doi: 10.1126/science.284.54 21.1805

Zeigerer, A., Lampson, M. A., Karylowski, O., Sabatini, D. D., Adesnik, M., Ren, M., et al. (2002). GLUT4 retention in adipocytes requires two 
intracellular insulin-regulated transport steps. Mol. Biol. Cell 13, 2421-2435. doi: 10.1091/mbc.e02-02-0071

Zerial, M., and Mcbride, H. (2001). Rab proteins as membrane organizers. Nat. Rev. Mol. Cell Biol. 2, 107-117. doi: 10.1038/35052055

Zhou, Z., Liu, A., Xia, S., Leung, C., Qi, J., Meng, Y., et al. (2018). The C-terminal tails of endogenous GluA1 and GluA2 differentially contribute to hippocampal synaptic plasticity and learning. Nat. Neurosci. 21, 50-62. doi: 10.1038/s41593017-0030-Z

Zhu, J. J., Esteban, J. A., Hayashi, Y., and Malinow, R. (2000). Postnatal synaptic potentiation: delivery of GluR4-containing AMPA receptors by spontaneous activity. Nat. Neurosci. 3, 1098-1106. doi: 10.1038/80614
Conflict of Interest Statement: The author declares that the research was conducted in the absence of any commercial or financial relationships that could be construed as a potential conflict of interest.

Copyright $\odot 2018$ Park. This is an open-access article distributed under the terms of the Creative Commons Attribution License (CC BY). The use, distribution or reproduction in other forums is permitted, provided the original author(s) and the copyright owner(s) are credited and that the original publication in this journal is cited, in accordance with accepted academic practice. No use, distribution or reproduction is permitted which does not comply with these terms. 